\title{
Guidelines for the diagnosis and management of osteoporosis in Poland. Update 2017
}

\author{
Roman Lorenc ${ }^{1}$, Piotr Głuszko ${ }^{2 *}$, Edward Franek ${ }^{3 *}$, , Mirosław Jabłoński ${ }^{4}$, Maciej Jaworski ${ }^{5}$, \\ Ewa Kalinka-Warzocha ${ }^{6 * * *}$, Elżbieta Karczmarewicz ${ }^{5}$, Tomasz Kostka ${ }^{7}$, Krystyna Księżopolska- \\ -Orłowska ${ }^{8}$, Ewa Marcinowska-Suchowierska ${ }^{9}$, Waldemar Misiorowski ${ }^{10 * * *}$, Andrzej Więcek $^{11}$
}

\author{
Poland \\ Poland \\ *speakers fee from Roche, Amgen, Novartis \\ ** speakers fee from Amgen \\ *** consulting fee Amgen \\ ****speakers fee Merck
}

${ }^{1}$ Multidisciplinary Osteoporosis Forum, SOMED, Warsaw, Poland

${ }^{2}$ Department of Rheumatology, National Institute of Geriatrics, Rheumatology and Rehabilitation, Warsaw, Poland

${ }^{3}$ Mossakowski Medical Research Centre, Polish Academy of Sciences, Warsaw, Poland

${ }^{4}$ Department of Rehabilitation and Orthopedics, Medical University of Lublin, Poland

${ }^{5}$ Department of Biochemistry, Radioimmunology and Experimental Medicine, The Childrens Memorial Health Institute, Warsaw,

${ }^{6}$ Department of Surgical Oncology and Breast Disease, Polish Mother's Memorial Hospital Research Institute, Lodz, Poland

${ }^{7}$ Department of Geriatrics, Medical University of Lodz, Healthy Ageing Research Centre (HARC), Central Veterans' Hospital, Lodz,

${ }^{8}$ Department of Rehabilitation, National Institute of Geriatrics, Rheumatology and Rehabilitation, Warsaw, Poland

${ }^{9}$ Department of Geriatrics, Internal Medicine and Metabolic Bone Diseases, Postgraduate Medical Education Center, Warsaw, Poland

${ }^{10}$ Department of Endocrinology, Postgraduate Medical Education Center, Warsaw, Poland

${ }^{11}$ Department of Nephrology, Transplantation and Internal Medicine, Medical University of Silesia, Katowice, Poland

\begin{abstract}
In the rapidly ageing society in Poland, osteoporosis is a growing epidemiological problem, and osteoporosis-related fractures are a cause of chronic disability and considerable increase of death risk. It turns out that 80 to $90 \%$ of patients suffering from osteoporosis, including osteoporosis accompanied by fractures, do not receive adequate pharmacotherapy. In this paper, a Guideline Working Group of experts from the Multidisciplinary Osteoporosis Forum update the existing Polish guidelines concerning the diagnosis and management of osteoporosis (last revised in 2013), taking account of the latest literature, availability and reimbursement of drugs, and current health care organisation. In the revised guidelines, we still postulate that tasks are divided between primary care doctors (stage I) and specialists in osteoporosis management (stage II). We emphasise the necessity of early initiation of pharmacotherapy and rehabilitation in all patients with low-energy fractures. We recommend that the 10-year fracture risk should be estimated in all patients (including those without fractures) who are over 50 years of age, and that the Polish threshold for therapeutic intervention should be adopted: $\geq 10 \%$ for FRAX PL calculator. We add strategies of drug choice and therapy monitoring with imaging, and densitometric and biochemical diagnostics. We define basic guidelines concerning prevention of falls, rehabilitation, and dietary procedures, and elimination of environmental and other fracture risk factors. We point to two vital elements for improving osteoporosis management: 1) strategy of supervision over fractures management - Fracture Liaison Service (FLS), and, optimally, 2) strategies of short-term monitoring of the therapeutic efficacy with the use of biochemical markers. (Endokrynol Pol 2017; 68 (5): 604-609)
\end{abstract}

Key words: osteoporosis diagnosis, prevention, treatment, rehabilitation

\section{Introduction}

More than 2 million people within the Polish population at the age of over 50 years (every third woman and every fifth man) suffer from osteoporosis, in most cases - complicated with fractures [1]. In spite of increased mortality, worse quality of life, and higher risk of subsequent fractures following the first fracture, only
10 to $20 \%$ of such patients receive pharmacological treatment. The number of patients with a high risk of the first fracture, who receive pharmacological treatment, is on a similar, unacceptably low level [2].

The purpose of this paper is to update and complete the existing Polish guidelines $[3,4]$ concerning the diagnosis and management of osteoporotic patients with or without history of fractures, with the aim of 
improving the existing status of underestimating the present osteoporosis-related threats.

\section{Methods}

A group of experts (specialists in rheumatology, orthopaedics and traumatology, internal medicine, geriatrics, endocrinology, rehabilitation, nephrology, diabetology, oncology, biochemistry, and imaging techniques) from the Multidisciplinary Osteoporosis Forum performed analysis of English-language publications in MEDLINE and COCHRANE databases concerning diagnosis, prevention, and therapeutic procedures, published from 2012 to March 2017. The agreed recommendations were preceded by abundant electronic correspondence and comments in writing. The task of the group was to update the "Guidelines" published in 2013 [3, 4]. The guidelines were rated by experts who voted using a 1-10 (10 = strongest) point scale to assess the "strength" of each recommendation in the Polish conditions. The existing guidelines were referred for review and accepted by national consultants in endocrinology, orthopaedics and traumatology, geriatrics, rheumatology, internal medicine, medical rehabilitation, diabetology, family medicine, and public health.

\section{Management strategy}

The general strategy is based on comprehensive diagnostic and therapeutic management, including division of tasks between primary care doctors at stage I and specialists treating osteoporosis at stage II.

\section{First stage}

All patients with suspected osteoporosis should be subject to selective screening including physical examination and history-taking, taking account of measurements of height and muscle strength, and assessment of clinical factors of fracture risk [including FRAX PL (http://www.shef.ac.uk/FRAX/tool.aspx?country=40), falls risk factors, and others]. Values $<10 \%$ of the 10 year fracture risk qualify for preventive action or further diagnostic procedures, depending on the patient's condition. Ten-year risk $\geq 10 \%$ of fractures in major locations, or $\geq 3 \%$ concerning neck of the femoral bone, qualify the patient for further osteoporosis diagnosis and comprehensive treatment in a specialist centre (stage II) [4].

Major fracture locations include: proximal femur (hip), vertebrae, proximal humerus, pelvis, distal radial bone $[2,4,5]$.

It should be stressed that each previous lowenergy fracture in the hip region and in other major locations highly increases the risk of subsequent fractures and is an absolute indication for fast initiation of comprehensive treatment (orthopaedic, pharmacological, analgesic, rehabilitation, and dietary interventions) [6].

\section{Second stage}

Specialist stage, includes differential diagnostics (secondary osteoporosis, other bone diseases, comorbidities) and detailed assessment of all fracture risks, including sarcopenia, for their possible elimination and for patient education. The specialist's task is to choose an effective pharmacotherapy based on densitometry (dual-energy X-ray absorptiometry - DXA) measurements, serum Vitamin D [25(OH)D] levels, together with calcium balance assessment and, possibly, bone turnover markers.

A fundamental role in choosing medication is played by in-label indications as well as contraindications, reimbursement options, and patient preferences [7]. It is also important to decide on adjuvant treatment, rehabilitation, fall prevention, calcium and vitamin D supplementation, diet modification, and a programme of therapy monitoring (Fig. 1).

In the case of a patient without low-energy fractures, pharmacotherapy is initiated by a specialist if $a \geq 10 \%$ fracture risk in major locations is confirmed in the 10-year perspective (calculated by FRAX BMD) and/or T-score upon DXA is $\leq-2.5 \mathrm{SD}$ regarding hip or lumbar spine.

In the case of low-energy fractures, orthopaedic treatment should be accompanied by pharmacological treatment, followed by rehabilitation. It is always recommended that DXA and detailed diagnostic procedures are performed in order to exclude or confirm secondary osteoporosis.

In the course of treatment, it is recommended that the efficacy and safety of therapy is regularly monitored $[8,9]$, and that the already existing and possibly occurring new fracture risk factors are periodically verified. Control of the patient's adherence to therapy, assessment of therapy efficacy, decision on a drug holiday, or drug switching depend on applied medication [8, 10], periodic densitometric examination, optimally, assessment of bone markers and X-ray examination, e.g. when the patient's height decreases significantly. In secondary osteoporoses, applied procedures should be subject to the requirements of the underlying disease management [9].

In patients with a lower fracture risk (fracture risk assessed by FRAX PL $\leq 5 \%$ ), it is recommended that dietary and lifestyle prevention measures are initiated $[4,5,7]$. 


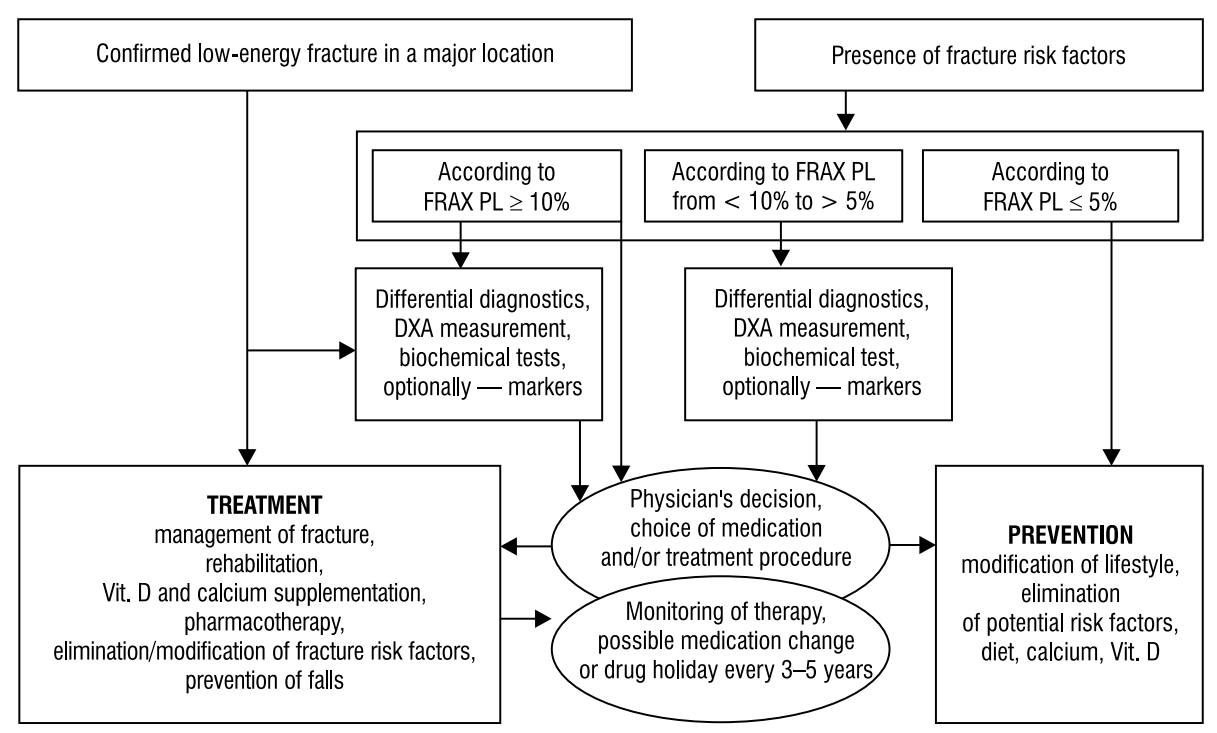

Figure 1. Overall algorithm of diagnostic and therapeutic procedures in postmenopausal and senile osteoporosis

\section{Guidelines}

1. We postulate that low-energy hip fractures in women over 50 years of age and men over 65 years of age, as well as every previous low-energy fracture in other major locations (after excluding other causes), with already existing osteopaenia (DXA, T-score < -1.5), is an obvious basis for diagnosing osteoporosis, and an indication for early initiation of comprehensive treatment (orthopaedic, pharmacological, analgesic, rehabilitation, and dietary) ["strength" 8.8].

2. We recommend, that immediately after surgical or conservative treatment of a low-energy fracture, the orthopaedic surgeon (traumatologist) initiates either oral or parenteral pharmacotherapy, or refers the patient to a competent osteoporosis therapy centre, in order to reduce the risk of subsequent fractures [9.1].

3. In patients of both sexes over 50 years of age, a high fracture risk in 10-year perspective (FRAX $\mathrm{BMD} \geq 10 \%$ ) or T-score $\leq-2.5$ for hip or vertebrae L1-L4 is also the basis for diagnosing osteoporosis, and for initiation of treatment [8.1]

4. We postulate that the primary care doctor and/ /or family doctor (stage I), based on physical examination (including height measurement), contributory history of fractures and falls, and fracture risk assessment by means of FRAX BMI for the Polish population, is entitled to: identify patients at risk of fractures and refer them to an outpatient osteoporosis clinic or other specialist engaged in osteoporosis management. For patients with a low fracture risk and without past fractures, the main task of the primary care doctor is to carry out preventive measures and patient education [7.5].

5. Tasks of the primary care doctor and/or family doctor should include continuation and monitoring of treatment initiated by the outpatient osteoporosis clinic or by other specialists, verification of possible adverse effects of drugs, and identification and, possibly, elimination of new fracture risk factors [7.3].

6. We recommend that the tasks of the specialist treating osteoporosis (stage II) should include confirmation of occurred fractures, identification of other existing fracture risks, and an ultimate decision on comprehensive therapy based on differential diagnosis, densitometry, bone turnover (optimally), determination of calcium-phosphate balance including 24-hour urinary calcium, and serum vitamin D levels [8.3].

7. Densitometry (DXA) remains the "gold standard" in osteoporosis diagnosis. The World Health Organisation (WHO) defines the following densitometric criteria for diagnosing osteoporosis on the basis of BMD measurement by means of DXA of the femoral neck (or spine) in postmenopausal women (T-score is expressed as the number of standard deviations from the reference point, which is the peak bone mineral density).

- 1) T score $>-1$ SD - normal value,

- 2) T score from -1 to $-2.5 \mathrm{SD}$ - osteopaenia,

-3) T score $\leq-2.5 \mathrm{SD}$ - osteoporosis,

-4) T score $\leq-2.5$ SD plus an osteoporotic fracture - advanced osteoporosis. 
Similar criteria have been adopted also for men $>50$ years of age [8.1].

8. In densitometry-based diagnostics in younger patients ( $<40$ years old), the Z-score should be taken into account, while the final diagnosis, assessment of fracture risk factors (FRAX is not applied in persons $<45$ years old), and the choice of therapy should be decided by the specialist in cooperation with the patient [8.6].

9. Current guidelines concerning harmonisation, standardisation, and automation of bone markers restrict the applied markers to CTX (C-terminal telopeptide of type I collagen) as a bone resorption marker, and PINP (N-terminal propeptide of type I procollagen) as a bone formation marker. Marker measurement is useful in therapy monitoring, "drug holidays", assessment of fracture risks, and in suspicion of malignancies. Postulates for marker testing and interpretation are defined by adequate GLP requirements [7.4].

10. An important role in diagnostics and therapy is played by the assessment of the patient's general condition, particularly of his/her musculosceletal system and condition of muscles (sarcopenia, myopathies, frailty syndrome, falls risk), evaluated mainly by means of measuring the muscle mass, walking speed, handgrip strength, and get up and go test [9.1].

11. Choice of a drug (stage II) of the highest antifracture effectiveness, individually for the patient, should be made by a specialist on the basis of BMD measurement and biochemical test results, taking account of in-label indications, possible contraindications, and therapy costs.

The specialist's tasks also include: definition of a therapy monitoring protocol, patient's education, and elimination of modifiable fracture risk factors [8.8 ].

12. Osteoporosis diagnostics and treatment in men of over 50 years of age does not differ substantially from those in postmenopausal women. Similar drugs are used for treatment, although some of them have not been approved for use in men (e.g. ibandronate, raloxifene). Administration of testosterone in men with deficiency of the hormone does improve the BMD result and general performance status, but the fracture risk-reducing effect of such therapy has not been proven [8.8].

13A. Bisphosphonates are the most commonly used anti-resorptive drugs, with the best established anti-fracture effect regarding spine, hip, and other non-vertebral locations. Efficacy of bisphosphonate therapy depends on prior obtaining and maintenance of normal serum 25(OH) D levels and calcium-phosphate balance [8.1].

13B. As well as regular yearly monitoring of therapy (BMD measurement in the same location by means of the same device), after three years of intravenous bisphosphonate administration, or after five years of oral bisphosphonate administration, it is necessary to verify the foregoing efficacy, safety, and possible purpose of further therapy, to consider the need of drug modification or drug holiday. Efficacy of medication can be evaluated after three months on the basis of bone marker measurements and comparison of the results to baseline values. In a longer perspective, it can be evaluated by BMD measurement [8.8].

13C. Therapy with bisphosphonate(s) in therapeutic doses (alendronate, risedronate, ibandronate, zoledronate) is considered to have failed if a new fracture occurs after 12 months of the therapy, or if the BMD value decreases after 12 months of the therapy in comparison to the baseline and the decrease is greater than the least significant change as measured in the same location [8.4].

14. Denosumab is an antibody inhibiting the maturation and function of osteoclasts, and it can be used as a first-line drug or when other therapies have been unsuccessful in osteoporotic patients of both sexes. It can be administered in sequential therapy after an anabolic; it can be also given to patients with severe renal failure. Its anti-resorptive effect is reversible, and the fracture risk may increase when the drug is discontinued; therefore, drug holidays are not recommended, and where it is necessary to stop the therapy, the patient should be prescribed any other anti-resorptive drug [8.5].

15. Despite of their established anti-fracture effect, also in severe secondary osteoporoses, parathyroid hormone $(\mathrm{PTH})$ derivatives, e.g. teriparatide (anabolic drugs), are rarely used because of their high cost. When the drug is discontinued (duration of therapy is limited to 24 months), treatment should be continued with the use of another anti-resorptive drug (bisphosphonate, denosumab) as a sequential therapy. Administration of PTH derivatives immediately after anti-resorptive drugs is not recommended [8.9].

16. Although it reduces the fracture risk, hormone replacement therapy (HRT — oestrogen-progestagen) in postmenopausal women is currently not recommended for the treatment of osteoporosis. Similarly, drugs from the SERM class (e.g. raloxifene), which reduce the risk of vertebral fractures, are used to a limited extent due to their adverse effects [8.2]. 
Table I. Anti-fracture efficacy of osteoporosis treatments (according to Reginster JY et al. Drugs 2011; 71: 65-78)

\begin{tabular}{|c|c|c|c|c|c|c|c|}
\hline & \multirow[t]{2}{*}{ FORM/DOSE } & \multirow[t]{2}{*}{ DOSAGE } & \multicolumn{3}{|c|}{ POSTMENOPAUSAL OSTEOPOROSIS } & \multirow{2}{*}{$\begin{array}{l}\text { MALE } \\
\text { OSTEOPOROSIS }\end{array}$} & \multirow[t]{2}{*}{ GIO } \\
\hline & & & $\begin{array}{l}\text { REDUCTION } \\
\text { OF } \\
\text { VERTEBRAL } \\
\text { FRACTURES }\end{array}$ & $\begin{array}{l}\text { REDUCTION } \\
\text { OF HIP } \\
\text { FRACTURES }\end{array}$ & $\begin{array}{l}\text { REDUCTION } \\
\text { OF NON- } \\
\text { VERTEBRAL } \\
\text { FRACURES }\end{array}$ & & \\
\hline ALENDRONATE & Tablets 70 mg & orally 1 × per week & YES & YES & YES & YES & YES \\
\hline \multirow[t]{2}{*}{ IBANDRONATE } & Tablets $150 \mathrm{~g}$ & orally $1 \times$ per month & YES & NO & NO & NO & NO \\
\hline & $\begin{array}{l}\text { Pre-filled syringe } \\
3 \mathrm{mg} / 3 \mathrm{ml}\end{array}$ & $\begin{array}{l}\text { i.v. } 1 \times \text { every three } \\
\text { months }\end{array}$ & & & & & \\
\hline RISEDRONATE & Tablets 35 mg & orally 1 × per week & YES & YES & YES & YES & YES \\
\hline ZOLEDRONATE & $\begin{array}{l}\text { Solution } 5 \mathrm{mg} / \\
/ 100 \mathrm{ml}\end{array}$ & i.v. $1 \times$ per year & YES & YES & YES & YES & YES \\
\hline DENOSUMAB & $\begin{array}{l}\text { Pre-filled syringe } 60 \\
\mathrm{mg} / 1 \mathrm{ml}\end{array}$ & $\begin{array}{l}\text { s.c. } 1 \times \text { every six } \\
\text { months }\end{array}$ & YES & YES & YES & YES & NO \\
\hline RALOXIFENE & Tablets 60 mg & orally $1 \times$ daily & YES & NO & NO & NO & NO \\
\hline TERIPARATIDE* & $\begin{array}{l}\text { Solution } 2 \mu \mathrm{g} / \\
/ 80 \mu \mathrm{l} \text { - pen } 3 \mathrm{ml}\end{array}$ & s.c. $1 \times$ daily & YES & NO & YES & YES & YES \\
\hline $\begin{array}{l}\text { STRONTIUM } \\
\text { RANELATE* }\end{array}$ & $\begin{array}{l}\text { Granulate for oral } \\
\text { suspension } 2 \mathrm{~g}\end{array}$ & orally $1 \times$ per daily & YES & YES & YES & YES & NO \\
\hline
\end{tabular}

17. Strontium ranelate is a dual-action drug, with an established anti-fracture effect in all locations. It is efficient in patients of both sexes with osteopaenia, osteoporosis, and in the oldest patients $(>80$ years of age). Due to possible thromboembolic complications and increased risk of cardiovascular diseases, the European Medicines Agency (EMA) imposed limits in its use [8.4].

18. An important role in the prevention and treatment of osteoporosis is played by optimal (diet and/ /or supplements) intake of calcium (about 1200 $\mathrm{mg} /$ day), protein $(1.2 \mathrm{~g} / \mathrm{kg}$ of body weight /day), potassium (about $3500 \mathrm{mg} /$ day), and magnesium (> $300 \mathrm{mg} /$ day). The necessary component of osteoporosis prevention and treatment is an adequate Vitamin D supply (minimum 800 IU/day), while the efficacy of pharmacotherapy depends on optimal serum levels of $25(\mathrm{OH}) \mathrm{D}$, and compensation of the calcium-phosphate balance [9].

19. In the case of secondary osteoporoses, it is vital to effectively manage the underlying disease. Considering the complexity of individual diseases, we recommend individualised management, possibly with reduction or elimination of osteoporosis-causing drugs, supplementation of nutrition deficiencies, kinesiotherapy, and elimination of additional fracture risk factors [8.8].

20. An especially common iatrogenic secondary osteoporosis is glucocorticoid-induced osteoporosis (GIO) caused by chronic administration of glucocorticoids. We recommend that fracture risk should be determined and followed by preventive actions or therapy in all patients receiving prednisone (or equivalent) in a daily dose $>2.5 \mathrm{mg}$ for $>3$ months. Preventive administration of bisphosphonate should be considered in patients over 50 years old with fracture risk factors, who receive $>5 \mathrm{mg}$ /day prednisone for $>3$ months, while such a procedure should be initiated obligatorily in patients over 65 years of age, receiving $\geq 7.5 \mathrm{mg} /$ day, even if they present no other fracture risk factors. A previous fracture is always an indication to initiate therapy [8.8].

21A. Fracture risk is higher in diabetic patients. In the case of type 1 diabetes, densitometric examination should be performed after five years from the disease diagnosis, and repeated every two to five years, depending on its results. In type 2 diabetes, the fracture risk may not correspond with the BMD value [8.1].

21B. The FRAX calculator is not suited for assessment of the fracture risk in young patients with type 1 diabetes; it can be more useful with older patients with type 2 diabetes, but the usefulness of the tool in such patients has not been verified [8.2].

22. Osteoporosis secondary to endocrinopathies, hyper- and hypothyroidism, hyperparathyroidism, diseases of adrenal cortex and hypothalamic-pituitary axis, and hypogonadism requires prior specialist endocrinological treatment of the underlying disease [8.8]. 
23. Due to the complex nature of calcium-phosphate metabolism disorders and diversity of bone lesion forms in each type of renal osteodystrophy, management of patients with chronic kidney disease (CKD), including dialysed patients, requires broad biochemical diagnostics and, in some cases, bone biopsy with histomorphometric assessment. CKD patients should be diagnosed and attended in specialist centres only [8.5].

24. A special group comprises patients with chronic gastrointestinal diseases, patients after bariatric surgeries, anorexics, persons following extreme vegan diets, etc. In such cases, both gastroenterological treatment and a dietician's (and sometimes a psychologist's) co-operation are essential [8.6].

25. An important component of patient care at all stages of osteoporosis treatment is comprehensive rehabilitation and prevention of falls [8.8].

26. Patients with moderate risk of fractures (10-5\%) may need further evaluation. Prevention of fractures should involve all patients, including those with a low fracture risk $(<5 \%)$. A healthy, active lifestyle, elimination of smoking and alcohol abuse, proper diet, reduction of the use of drugs that increase fracture risk, prevention of falls and injuries, and adequate social education are fundamental conditions for the risk reduction of osteoporosis and its complications [9.3].

\section{References}

1. Svedbom A, Hernlund E, Ivergård M, et al. EU Review Panel of IOF Osteoporosis in the European Union: a compendium of country-specific reports. Arch Osteoporos. 2013; 8: 137, doi: 10.1007/s11657-013-0137-0, indexed in Pubmed: 24113838.

2. Marcinowska-Suchowierska E, Głuszko P, Badurski J, et al. Leczenie farmakologiczne osteoporozy w Polsce - dostępność, przyczyny braku jej wdrażania. Post Nauk Med. 2015; 12: 879-885. (in Polish).

3. Lorenc R, Głuszko P, Karczmarewicz E, et al. Zalecenia postępowania diagnostycznego i leczniczego w osteoporozie. Aktualizacja 2013. Medycyna Praktyczna, wyd. specj. Reumatologia. 2013; 1. (in Polish).

4. Głuszko P, Lorenc RS, Karczmarewicz E, et al. Working Group including the representatives of the Polish Associations of Orthopedics and Traumatology, Rehabilitation, Gerontology, Rheumatology, Family Medicine, Diabetology, Laboratory Diagnostics, Andropause and Menopause, Endocrinology, Radiology, and the STENKO group. Polish guidelines for the diagnosis and management of osteoporosis: a review of 2013 update. Pol Arch Med Wewn. 2014; 124(5): 255-263, indexed in Pubmed: 24694725.

5. Kanis JA, McCloskey EV, Johansson H, et al. Scientific Advisory Board of the European Society for Clinical and Economic Aspects of Osteoporosis and Osteoarthritis (ESCEO) and the Committee of Scientific Advisors of the International Osteoporosis Foundation (IOF). European guidance for the diagnosis and management of osteoporosis in postmenopausal women. Osteoporos Int. 2013; 24(1): 23-57, doi: 10.1007/s00198-0122074-y, indexed in Pubmed: 23079689.

6. Lems WF, Dreinhöfer KE, Bischoff-Ferrari H, et al. EULAR/EFORT recommendations for management of patients older than 50 years with a fragility fracture and prevention of subsequent fractures. Ann Rheum Dis. 2017; 76(5): 802-810, doi: 10.1136/annrheumdis-2016-210289, indexed in Pubmed: 28007756.

7. Cosman F, de Beur SJ, LeBoff MS, et al. National Osteoporosis Foundation. Clinician's Guide to Prevention and Treatment of Osteoporosis. Osteoporos Int. 2014; 25(10): 2359-2381, doi: 10.1007/s00198-014-2794-2, indexed in Pubmed: 25182228.

8. Adler RA, El-Hajj Fuleihan G, Bauer DC, et al. Managing Osteoporosis in Patients on Long-Term Bisphosphonate Treatment: Report of a Task Force of the American Society for Bone and Mineral Research. J Bone Miner Res. 2016; 31(1): 16-35, doi: 10.1002/jbmr.2708, indexed in Pubmed: 26350171.

9. Leszczynski P, Korkosz M, Pawlak-Bus K, et al. Diagnostyka i leczenie osteoporozy — zalecenia Polskiego Towarzystwa Reumatologicznego 2015. Forum Reumatologiczne. 2015; 1(1): 12-24. (in Polish).

10. McClung MR. Cancel the denosumab holiday. Osteoporos Int. 2016; 27(5): 1677-1682, doi: 10.1007/s00198-016-3553-3, indexed in Pubmed: 26932443. 